\title{
Design optimization of bidirectional arterial perfusion cannula
}

Saad Abdel-Sayed ${ }^{1 *}$ (D) Enrico Ferrari ${ }^{2}$, Philippe Abdel-Sayed ${ }^{3}$, Markus Wilhelm ${ }^{4}$, Ludwig-Karl von Segesser ${ }^{1}$ and Denis Berdajs 5

\begin{abstract}
Objectives: Determine if shortening the covered section of a self-expanding bidirectional arterial cannula, can enhance retrograde flow and thus reduce the risk of lower limb ischemia.
\end{abstract}

Methods: Outlet pressure vs flow rate was determined for three cannulas types: a $15 \mathrm{~F}$ self-expanding bidirectional cannula having a covered section of $90 \mathrm{~mm}$, the same cannula but with a shorter covered section of $60 \mathrm{~mm}$, and a Biomedicus cannula as control. The performances of all the cannulas were compared using a computerized flowbench with calibrated sensors and a centrifugal pump. Water retrograde flow was determined using a tank timer technique. Anterograde and retrograde flow rate versus outlet pressure were determined at six different pump speed.

Results: For each of the six pump speed, both bidirectional cannulas, 60-mm covered and 90-mm covered respectively, showed higher performance than Biomedicus cannula control, as demonstrated by higher flow rate and lower pressure. We also observed that for the bidirectional cannula with shorter covered section, i.e. $60 \mathrm{~mm}$ coverage, provides enhanced performance as compared to a 90-mm coverage. Finally, the flow rate and the corresponding pressure can be consistently measured by our experimental set-up with low variability.

Conclusions: The new configuration of a shorter covered section in a bidirectional self-expanding cannula design, may present an opportunity to overcome lower leg ischemia during extra-corporal life support with long term peripheral cannulation.

Keywords: Virtually wall-less cannula, Bidirectional cannula, Arterial cannulation, ECMO, ECLS, Leg ischemia

\section{Introduction}

Femoral arterial cannulation for arterial inflow is essential for cardiac surgical procedures, as well as for extracorporal life support (ECLS) and extra-corporal membrane oxygenation (ECMO). The longer the femoral artery perfusion, the higher the risk of serious problems for distal perfusion. An eventual obstruction of the access vessel by traditional rectilinear cannulas design may cause irreversible hindrances such as amputation or even death. Several authors have stated an important risk of lower limb

\footnotetext{
* Correspondence: saad.abdel-sayed@chuv.ch

${ }^{1}$ Department of Surgery and Anesthesiology, CHUV, Lausanne, Switzerland Full list of author information is available at the end of the article
}

ischemic hitches after protracted cannulation of femoral artery, such as Hendrickson and Glower, who reported a rate of $11.5 \%$ after peripheral CPB [1]. Also, Foley et al. [2] and Huang et al. [3] have demonstrated that ECMO support is correlated with lower limb ischemic problem at rates as high as $26 \%$.

Collateral blood flow is responsible for the viability of the lower limb. Incidence of lower limb ischemia is often related to deprived flow rate, leading to an obligation for fasciotomy or even amputation. A number of techniques have been proposed to prevent this potentially devastating complication, including the use of a downstream femoral perfusion catheter $[1,4-6]$ or an end-to-side femoral 
artery graft $[7,8]$. However, these techniques are often difficult to perform, and in addition to the complexity of the procedure, they are not always consistent. Besides, bleeding complications and high risk of infection are often related to these techniques [9]. Thus, a new femoral cannulation system is necessary for clinical surgery and standard retrograde perfusion without reducing distal limb blood flow.

To overcome these difficulties, Smartcanula ${ }^{\circ}$ LLC (Lausanne, Switzerland) developed a self-expandable bidirectional design for cannulation of femoral artery. This cannula has the enhanced performance of a nearly wallless design as compared to the traditional rectilinear percutaneous cannulas. Besides, this cannula expands partially, i.e. at the insertion position only with regard to the vessel lumen, allowing thus parallel retrograde flow as the cannula body does not occupy the whole vascular lumen [10]. This novel bidirectional design allowed for important high flow rates at lesser driving pressures. Here, we present an optimized configuration of this bidirectional arterial cannula. The optimization development consists in shortening the narrow covered section of the cannula, which we assume could enhance peripheral perfusion, and thus diminish the risk of lower limb ischemia and subsequent complications during ECLS with peripheral cannulation [7]. Furthermore, we hypothesize that reducing size of the covered section may result in early cannulation and enhanced comfort without reducing the quality of peripheral perfusion.

\section{Material and methods Experimental setup In vitro evaluation of forward flow rate and pressure}

Pressure values $(\mathrm{P})$ and cannula anterograde flow rates (Q) were measured for both self-expanding cannulas and the rectilinear design control cannula using a simple in vitro circuit, as previously described [10]. Briefly, the in vitro circuit consisted of a hard-shell reservoir with the water being pumped to the tested cannula with a centrifugal pump through a $1 \mathrm{~m}$ long silicone" $1 / 2$ " tubing (Fig. 1, upper panel). Flow and outlet pressure were determined at six pump speed of 500, 1000, 1500, 2000, 2500, and 3000 RPM, using a bench calibrated flowmeter and adjusted pressure sensors, as well as a data acquisition system and LabView application.

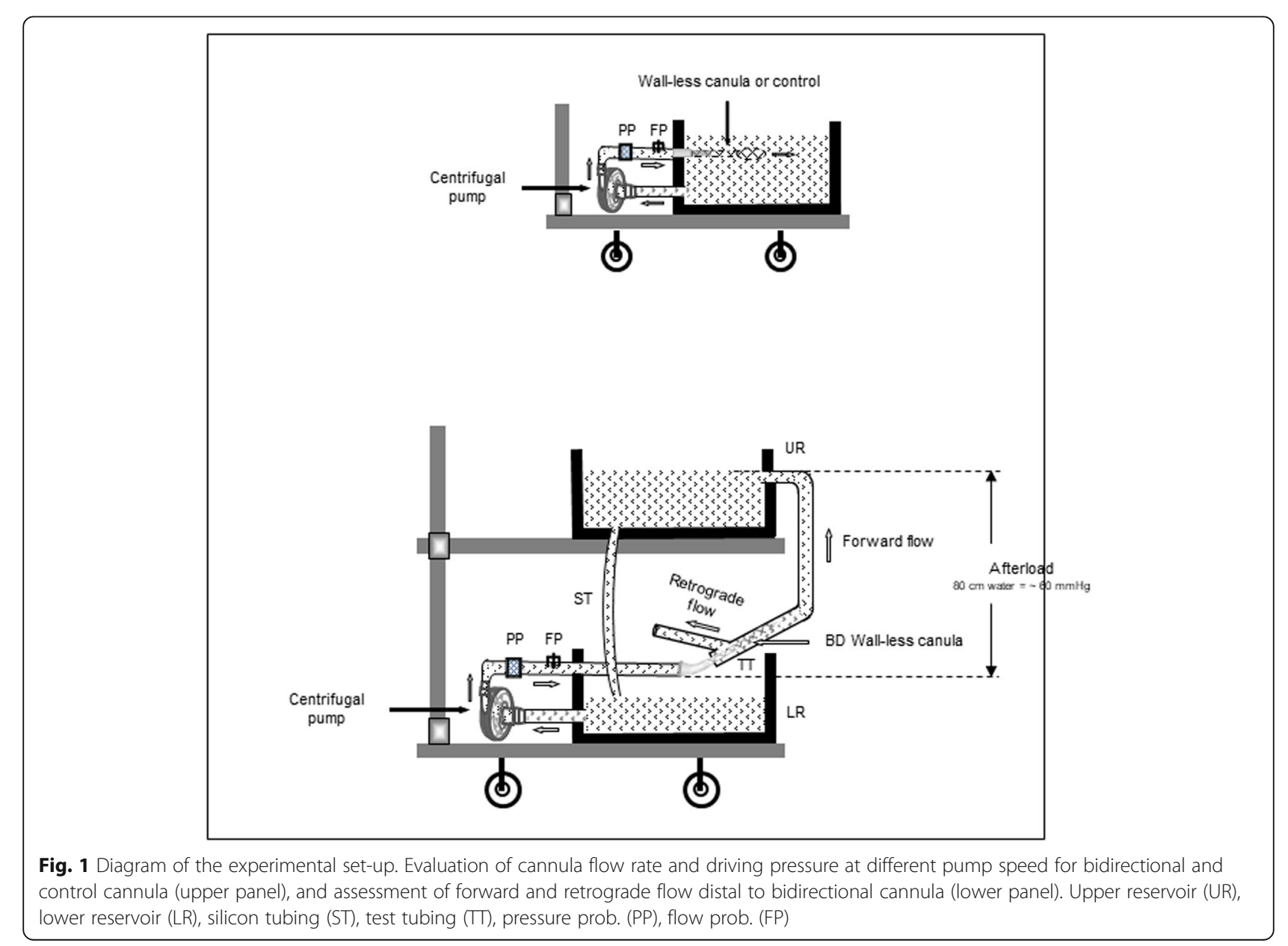




\section{Comparison of anterograde and retrograde flow distal to bidirectional cannula}

The second in vitro circuit, allowing to measure both anterograde and retrograde flows, included two hard-shell reservoirs, an upper reservoir (UR) connected to a lower reservoir of the same size (LR) by a silicone tubing (ST) to keep the afterload volume of water constant. The vertical distance between the LR and the UR was set to $80 \mathrm{~cm}$ to correspond to a pressure of approx. $60 \mathrm{mmHg}$.

LR was connected to a centrifugal pump and silicon tubing as described for the first set-up. Here, the difference in the second circuit, remains in the fact that the tested cannulas were inserted into a test tubing (TT) of $18 \mathrm{~F}$ diameter and $20 \mathrm{~cm}$ long, containing an orifice on one side, $2 \mathrm{~cm}$ far from its end (at the site of cannula insertion) allowing to measure the retrograde flow, and connected to the UR at its other end (Fig. 1, lower panel). Water was pumped from the LR to the UR through the TT representing the anterograde flow. The water retrograde flow was determined from the orifice of the TT using the tank timer technique. Both, anterograde and retrograde flow versus outlet pressure were determined for the six RPM mentioned above. Furthermore, gap between the cannula and the wall of the simulated vessel was measured.

\section{Cannulas}

Two smart bidirectional arterial cannula of $220 \mathrm{~mm}$ long were used in this study. As illustrated in Fig. 2, both cannulas have a meshed helical fusiform configuration, with respectively $90 \mathrm{~mm}$ and $60 \mathrm{~mm}$ long covered access section ( $15 \mathrm{~F}$ diameter for both), intended for non-occlusive cannula insertion, leading to a conical extra-corporeal "3/8" connecting sleeve. The uncovered fusiform section can be collapsed for a facilitated insertion and then be expanded to $24 \mathrm{~F}$ once inserted; in situ expansion allows free blood circulation in all directions (anterograde and retrograde), due to a free space between the bidirectional cannula and the artery wall, allowing for retrograde flow (Fig. 3). A third percutaneous Biomedicus arterial cannula (Medtronic, Tolochenaz, Switzerland) was used as a control for comparison.

\section{Statistics}

Each experimental measurement of flow rate and pressure was repeated six times for statistical analysis. Data were presented as means \pm standard of deviation (SD). Precision was determined by the coefficient of variation (CV), which was calculated by the SD / mean. The unpaired Student $\mathrm{t}$-test was used to compare the results of two cannulas. The standard two-way ANOVA test was used to compare between more than two cannulas. The significance level was $p<0.05$.

\section{Results}

\section{Comparison of anterograde flow rate versus pressure}

Self-expanding arterial cannulas, $60 \mathrm{~mm}$ and $90 \mathrm{~mm}$ covered access section, clearly demonstrated higher forward $\mathrm{Q}$ and lower $P$ values with regard to the control cannula (Fig. 4, upper panel). The forward flow rate steadily increased with the increase of pump speed (Fig. 4, lower panel). Improved anterograde flow rate to each revolution per minute for both self-expanding bidirectional cannulas 60 , was on average 15 and $9 \%$ respectively compared to the standard control cannula (Table 1).

\section{Comparison of retrograde flow rate versus pressure}

Results clearly show that the $60 \mathrm{~mm}$ covered access section bidirectional cannula provided higher retrograde flow rate as compared to the $90 \mathrm{~mm}$ covered. At 100 $\mathrm{mmHg}$ driving pressure, the corresponding retrograde flow rate values were $325 \pm 0.2$ and $200 \pm 2 \mathrm{~m} \mathrm{l} / \mathrm{min}$ for the $60 \mathrm{~mm}$ and $90 \mathrm{~mm}$ covered bidirectional cannulas, respectively (Fig. 5, upper panel). Retrograde flow rate steadily increased with the rise of pump speed. At 2000 RPM, retrograde flow rate was $309.50 \pm 46$ and $216.75 \pm$

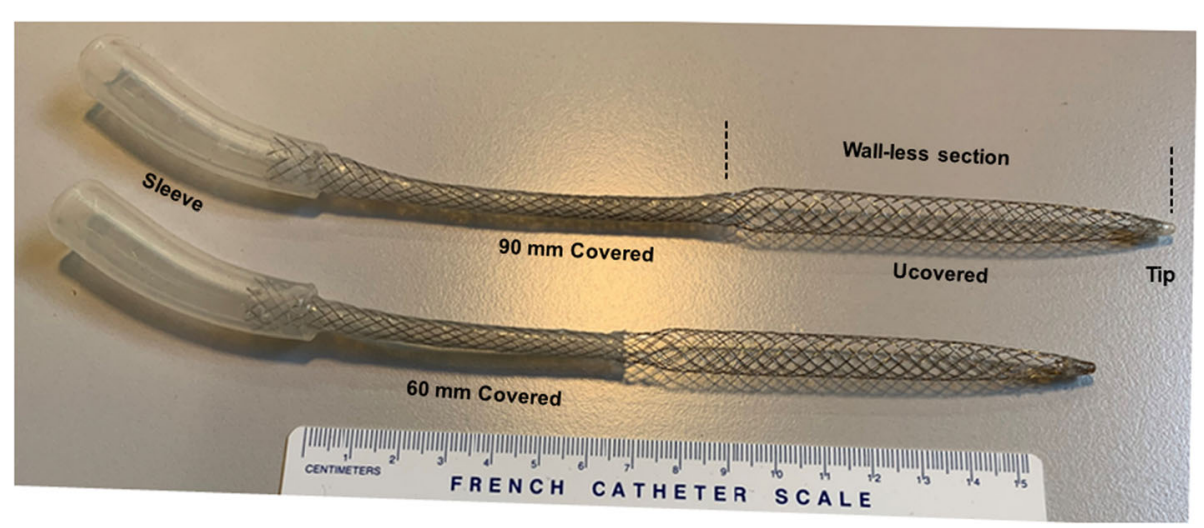

Fig. 2 Smart bidirectional perfusion cannula $90 \mathrm{~mm}$ covered (upper cannula), $60 \mathrm{~mm}$ covered (lower cannula) 


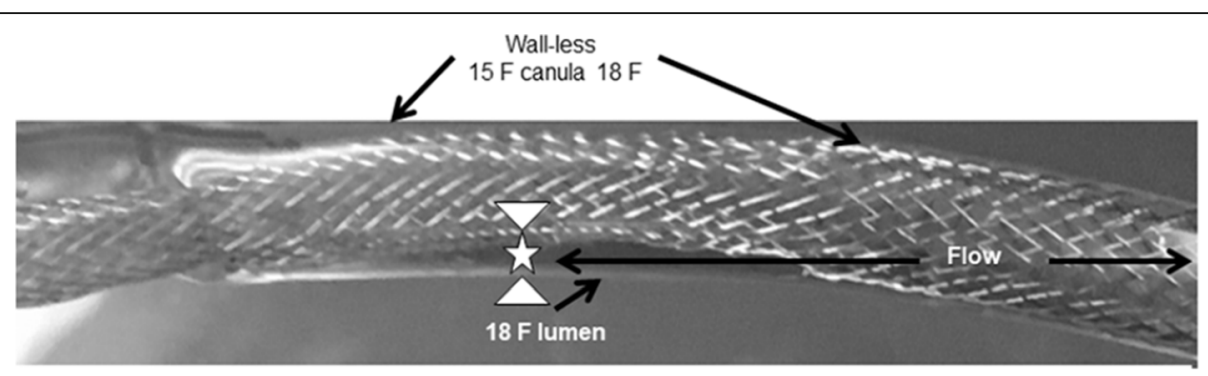

Fig. 3 Bidirectional Smartcanula inserted from the left to the right within a transparent tube mimicking femoral cannulation. The anterograde main flow intended for central perfusion is from the left to the right. A free space forms between the $15 \mathrm{~F}$ uncoated part of the cannula and the transparent tube. The fusiform uncovered section of the bidirectional cannula expands to the vessel wall $(18 \mathrm{~F})$ and provides retrograde flow to the periphery (limb)

$17 \mathrm{ml} / \mathrm{min}$ for the $60 \mathrm{~mm}$ and $90 \mathrm{~mm}$ covered respectively (Fig. 5, lower panel). Regular design arterial Biomedicus control with the performance closest to the test cannula did not show retrograde water flow. The residual lumen allowing for retrograde flow accounted for $9.81 \pm 0.83 \mathrm{~mm}^{2}$ for bidirectional versus non-measurable for control. The kinking assessment of self-expanding cannulas tests confirmed for all self-expanding cannula diameters $(12 \mathrm{~F}-36 \mathrm{~F})$, that the double helix design is extremely kink resistant $\left(180^{\circ}\right.$ and more) as shown in Fig. 6.

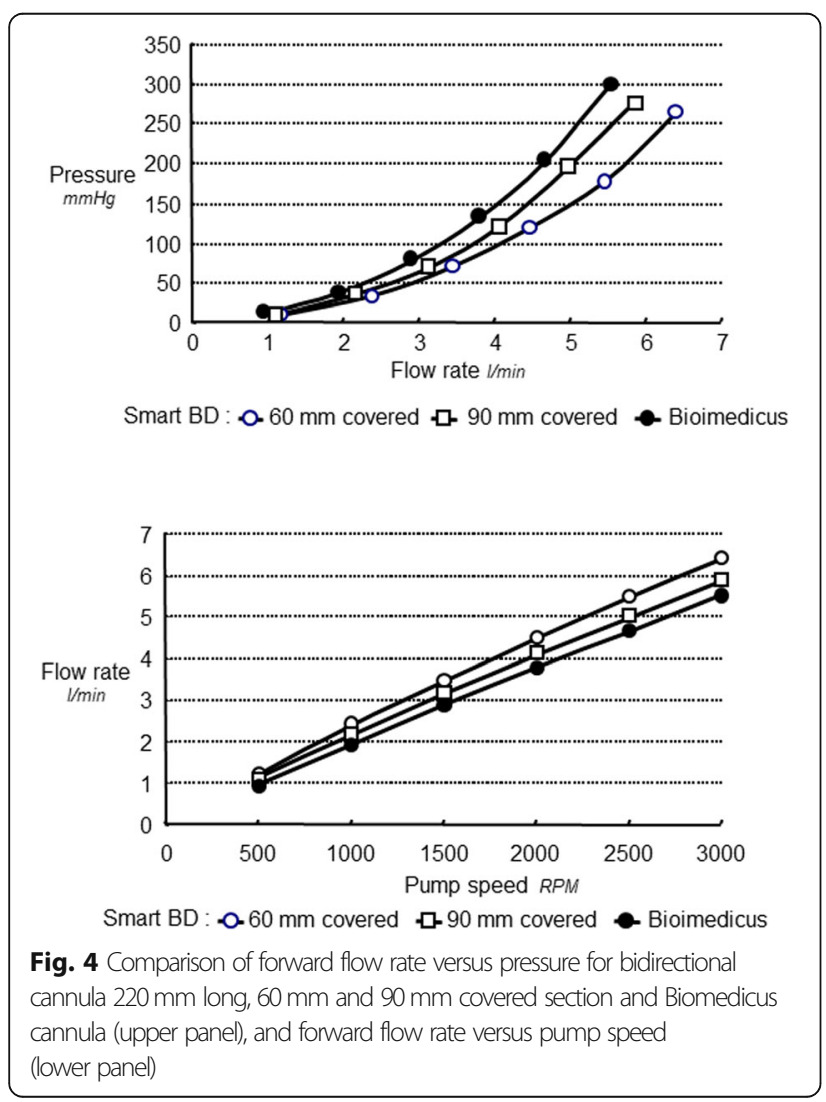

\section{Discussion}

Overall, both self-expanding bidirectional showed better forward flow rate to each revolution per minute when compared to the standard control cannula. This superior forward flow rate with the virtually wall-less cannulas is probably due to their tinny wall thickness $(0.36 \mathrm{~mm})$ as compared to the wall leanness of percutaneous cannula $(>0.60 \mathrm{~mm})$. This difference in wall thickness affects the effective cross-sectional area. The superiority of the bidirectional cannula was revealed with less positive pressure for each forward flow rate, compared with the regular control cannula (Fig. 4 , upper panel) $(p<0.001)$. In vivo perfusion with high positive pressure in rectilinear cannula design increases the risk of hemolysis and embolism formation.

Furthermore, the short bidirectional cannula design ( $60 \mathrm{~mm}$ of the $15 \mathrm{~F}$ covered access section), showed significantly higher forward flow rates at lower driving pressures as compared to the $90 \mathrm{~mm}$ covered. For a driving pressure of $100 \mathrm{mmHg}$, typical of a clinical situation, the corresponding flow rate is around $4.1 \mathrm{l} / \mathrm{min}$ for bidirectional $60 \mathrm{~mm}$ covered and versus $3.8 \mathrm{l} / \mathrm{min}$ for bidirectional cannula $90 \mathrm{~mm}$ covered, versus $3.2 \mathrm{l} / \mathrm{min}$ for control cannula corresponding to around $128 \%$ for 60 $\mathrm{mm}$ versus control and $108 \%$ for $60 \mathrm{~mm}$ versus $90 \mathrm{~mm}$. The advantages shown for the bidirectional $60 \mathrm{~mm}$ covered can be repeated at all pump speeds with minimal variations (CV between 1 and $4 \%$ for six repeated measures). Thus, shortening the $15 \mathrm{~F}$ free section length of the bidirectional cannula permits for higher flow rates at lower driving pressures. For a flow rate of $4.5 \mathrm{l} / \mathrm{min}$ the corresponding driving pressure is $180 \mathrm{mmHg}$ for the regular design control of the same diameter versus 150 $\mathrm{mmHg}$ for bidirectional cannula $90 \mathrm{~mm}$, versus 119 $\mathrm{mmHg}$ for bidirectional $60 \mathrm{~mm}$ covered, equal to around $150 \%$ for control versus $60 \mathrm{~mm}$ covered, and $126 \%$ for $90 \mathrm{~mm}$ covered versus $60 \mathrm{~mm}$ covered. (Fig. 4, upper panel). In addition, bidirectional $60 \mathrm{~mm}$ covered showed $160 \%$ more retrograde flow rate as compared to the 90 
Table 1 Comparison of flow rate and positive pressure at different pump speed

\begin{tabular}{llllllll}
\hline Cannula & RPM & $\mathbf{5 0 0}$ & $\mathbf{1 0 0 0}$ & $\mathbf{1 5 0 0}$ & $\mathbf{2 0 0 0}$ & $\mathbf{2 5 0 0}$ & $\mathbf{3 0 0 0}$ \\
\hline Biomedicus & Pressure $\mathrm{mmHg}$ & $10.27 \pm 1.01$ & $36.11 \pm 3,29$ & $76.45 \pm 3,16$ & $130.00 \pm 5,95$ & $201,30 \pm 15,12$ & $298,33 \pm 26,43$ \\
${ }^{\mathrm{a}} \mathrm{BD} 90 \mathrm{~mm}$ & & $8,86 \pm 0,15$ & $30,44 \pm 0,39$ & $68.25 \pm 14.86$ & $120,01 \pm 0,50$ & $194,93 \pm 0,33$ & $275,90 \pm 0,47$ \\
b $\mathrm{BD} 60 \mathrm{~mm}$ & & $8,35 \pm 0,40$ & $32.39 \pm 0.30$ & $68,62 \pm 0,88$ & $119,25 \pm 0,11$ & $180,00 \pm 18,34$ & $261,83 \pm 0,64$ \\
Biomedicus & Flow rate & $0.94 \pm 0.01$ & $1.93 \pm 0,01$ & $2,88 \pm 0,01$ & $3,77 \pm 0,01$ & $4.65 \pm 0,01$ & $5.52 \pm 0.01$ \\
a BD 90 mm & & $1,10 \pm 0,01$ & $2,15 \pm 0,01$ & $3,13 \pm 0,01$ & $4.05 \pm 0.01$ & $4,96 \pm 0,01$ & $5.85 \pm 0,01$ \\
bBD 60 mm & & $1.17 \pm 0,01$ & $2,37 \pm 0.01$ & $3,44 \pm 0,01$ & $4,46 \pm 0,01$ & $5.44 \pm 0,01$ & $6,38 \pm 0,01$ \\
\hline
\end{tabular}

Mean \pm SD; $n=6$

*** $p<0.0001$ for Bidirectional cannula versus Control cannula

aidirectional $90 \mathrm{~mm}$ covered

${ }^{b}$ Bidirectional $60 \mathrm{~mm}$ covered

mm covered. With our experimental system, the rectilinear design Biomedicus control did not show retrograde flow rate.

In clinical practice, a considerable bigger size of a traditional rectilinear percutaneous cannula would be needed to reach the same flow rate at the same driving pressure, due to less or no space for retrograde flow to the limb. Our results indicate that a considerable smaller cannula diameter of bidirectional design with $15 \mathrm{~F}$ covered access section can be used to attain a given target flow rate and a physiologic driving pressure.

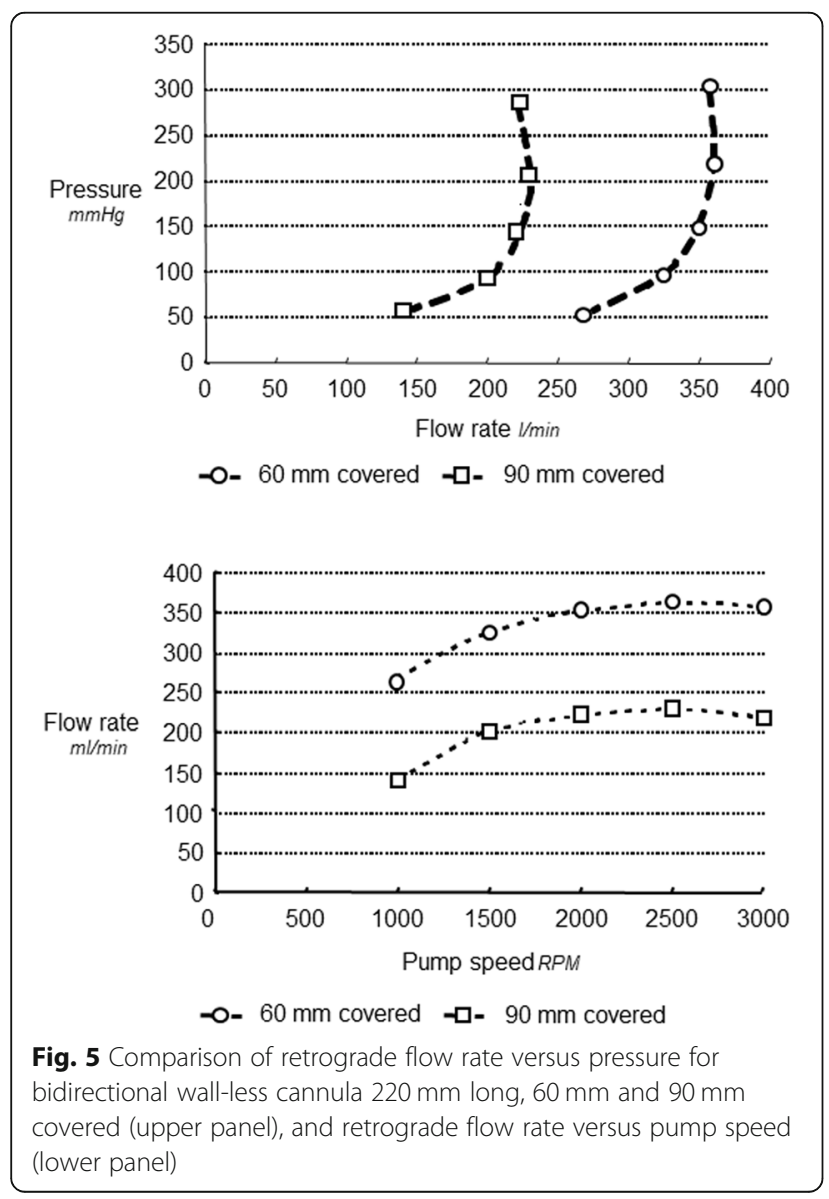

In the clinical setting, bidirectional cannula design is based on the "collapsed insertion and in situ expansion principle", which has been confirmed to be reliable [8]. Thus, for the clinical application no concerns about the insertion and the removal of a bidirectional cannula with its fusiform segment larger than the cannula diameter at the site of insertion [10] (Fig. 2). This function is achieved by extending the fusiform cannula section with a mandrel [11]. Similarly, the bidirectional canula can be removed easily, because it collapses with simple traction. Digital compression for caudal bleeding.

The results of this study were performed with water as the testing medium, which might be considered as a limitation when compared to tests performed with blood, for describing results relevant to the clinical setting. Thus, we questioned whether the outperformance of the cannula would be identical using blood instead of water, and if the higher viscosity of the blood would change its performance. Our preceding studies demonstrated, that the viscosity of the blood decreased flow by about 10 and $6 \%$ less for rectilinear percutaneous cannulas and virtually wall-less cannulas respectively [12], similar to the bidirectional design presented here. Also, Broman el. al $[13,14]$ showed that when blood with

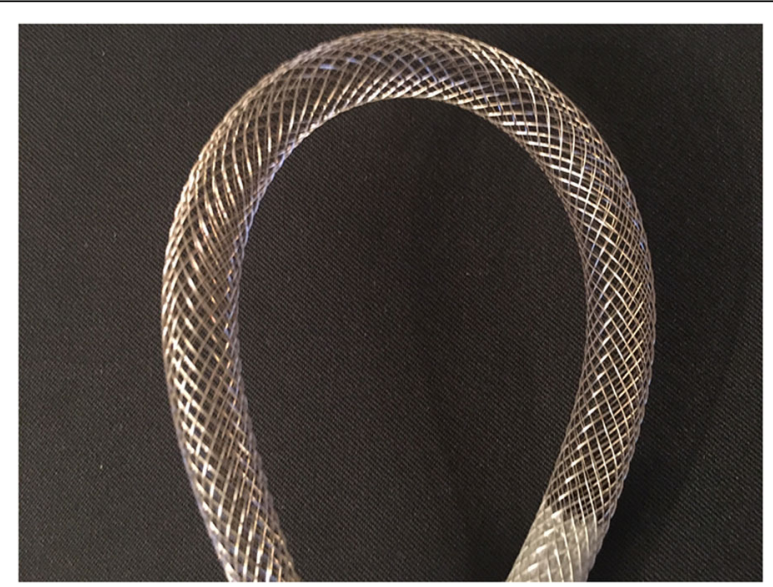

Fig. 6 Curved Smartcanula demonstrating its kink resistance. The latter is due to its double helix design 
hematocrit of $27 \%$ was used, the venous drainage pressure was steadily higher for a given flow than when water was used. Broman and co-authors demonstrated that blood flows with single-lumen return arterial cannulas for peripheral ECMO tested were lower than those provided by manufacturers using water. We think that water is a good testing medium for the cannula performance. The main advantage of water as test medium for cannula performance assessment is its reproducibility. This also explains why water is usually used as the industry standard medium for comparative to compare cannula performance validation. For the present set-up, the water transparency was necessary for assessment of the residual lumen resulting in retrograde flow.

Severe atheromatic disease access vessel or kinked access vessels may restrict the self-expanding mechanism of the bidirectional cannula and therefore the flow may also be delayed in one or both directions. The kinking assessment of self-expanding cannulas tests confirmed for all self-expanding cannula diameters $(12 \mathrm{~F}-36 \mathrm{~F})$, that the double helix design is extremely kink resistant $\left(180^{\circ}\right.$ and more) as shown in Fig. 6. However, a traditional rectilinear cannula with a fixed outer diameter may not even be inserted. The use of per-procedural ultrasound is a good tool in such complex situations as described above. The latter is helpful for target vessel identification and also guide-wire position clarification. For the bidirectional cannula, ultrasound allows in addition for identification of the flow direction by the means of the Duplex Color Doppler function [8]. We conclude bidirectional flow for ECLS and ECMO can be achieved on the arterial side with cannulas having a $15 \mathrm{~F}$ section, that does not occupy completely the access vessel for a retrograde flow, and which performance can be optimized by shortening its covered section. We consider that this type of cannula can also be used and optimized for MICS [15-17]. In vivo studies are planned as the next step for confirmation of these findings prior to clinical application.

\section{Conclusion}

The enhanced performance in a bidirectional self-expanding cannula with in a shorter covered section, may present an opportunity to overcome lower leg ischemia during ECLS with long term peripheral cannulation.

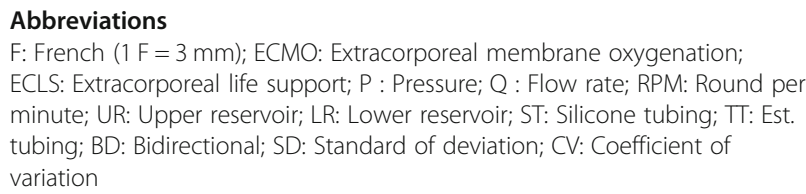

\section{Acknowledgements}

We would like to thank Smartcanula LLC (Lausanne, Switzerland) for providing the cannulas for testing free of charge.

\section{Our waiver request}

Has been granted.

Conflict of interest

Non declared.

\section{Authors' contributions}

Saad Abdel-Sayed. and L-K. von: Segesser designed the study. Saad AbdelSayed. and Phillippe. Abdel-Sayed performed the experiments. All authors contributed to the data analysis and manuscript writing. The author(s) read and approved the final manuscript.

\section{Funding}

Ludwig Karl von Segesser is founder of Smartcanula LLC. Smartcanula LLC Lausanne, Switzerland provided the samples for testing free of charge. No further funding was available for this study.

Availability of data and materials

All data generated or analyzed during this study are included in this published article and its supplementary information files.

\section{Declarations}

Ethics approval and consent to participate

This study did not require the use of any animal models or human subjects, and therefore no ethics approval or informed consent were needed.

\section{Consent for publication}

This study did not involve any individual, and did not contain any personal data related to any individual, therefore no consent for publication was required.

\section{Competing interests}

Ludwig Karl von Segesser is founder of Smartcanula LLC.

\section{Author details}

${ }^{1}$ Department of Surgery and Anesthesiology, CHUV, Lausanne, Switzerland. ${ }^{2}$ CardioCentro Ticino, Lugano, Switzerland. ${ }^{3}$ Department of Musculoskeletal Medicine, CHUV, Lausanne, Switzerland. ${ }^{4}$ Cardio-Vascular Surgery, Zurich University Hospital, Zurich, Switzerland. ${ }^{5}$ Division of Cardiac Surgery, University Hospital, Basel, Switzerland.

Received: 7 December 2020 Accepted: 13 April 2021

Published online: 26 April 2021

References

1. Hendrickson SC, Glower DD. A method for perfusion of the leg during cardiopulmonary bypass via femoral cannulation. Ann Thorac Surg. 1998; 65(6):1807-8. https://doi.org/10.1016/S0003-4975(98)00302-6.

2. Foley PJ, Morris RJ, Woo EY, Acker MA, Wang GJ, Fairman RM, et al. Limb ischemia during femoral cannulation for cardiopulmonary support. J Vasc Surg. 2010;52(4):850-3. https://doi.org/10.1016/j.jvs.2010.05.012.

3. Huang SC, Yu HY, Ko WJ, Chen YS. Pressure criterion for placement of distal perfusion catheter to prevent limb ischemia during adult extracorporeal life support. J Thorac Cardiovasc Surg. 2004;128(5):776-7. https://doi.org/10.101 6/j.jtcvs.2004.03.042

4. Greason KL, Hemp JR, Maxwell JM, Fetter JE, Moreno-Cabral RJ. Prevention of distal limb ischemia during cardiopulmonary support via femoral cannulation. Ann Thorac Surg. 1995;60(1):209-10. https://doi.org/10.1016/ S0003-4975(95)00380-0.

5. Kasirajan V, Simmons I, King J, Shumaker MD, DeAnda A, Higgins RS. Technique to prevent limb ischemia during peripheral cannulation for extracorporeal membrane oxygenation. Perfusion. 2002;17(6):427-8. https:// doi.org/10.1191/0267659102pf614oa.

6. Yoshimura N, Ataka K, Nakagiri K, Azami T, Yoshida M, Yamashita C, et al. A simple technique for the prevention of lower limb ischemia during femoral veno-arterial cardiopulmonary support. J Cardiovasc Surg. 1996;37(6):557-9.

7. von Segesser L, Marinakis S, Berdajs D, Ferrari E, Wilhelm M, Maisano F. Prevention and therapy of leg ischaemia in extracorporeal life support and extracorporeal membrane oxygenation with peripheral cannulation. Swiss Med Wkly. 2016;146:W14304. 
8. von Segesser LK, Ferrari E, Delay D, Maunz O, Horisberger J, Tozzi P. Routine use of self-expanding venous cannulas for cardiopulmonary bypass: benefits and pitfalls in 100 consecutive cases. Eur J Cardiothorac Surg. 2008;34(3): 635-40. https://doi.org/10.1016/..ejcts.2008.05.037.

9. Chen Y, Tutungi E, McMillan J, Tayeh SM, Underwood JK, Wells AC, et al. Pressure and flow characteristics of a novel bidirectional cannula for cardiopulmonary bypass. Innovations. 2017;12(6):430-3. https://doi.org/10.1 097/imi.0000000000000424.

10. Abdel-Sayed S, Ferrari E, Abdel-Sayed P, Wilhelm M, Halbe M, von Segesser LK, et al. New bidirectional arterial perfusion device. Int J Artif Organs. https://doi.org/10.1177/039139882090184.

11. Mueller XM, Mallabiabrena I, Mucciolo G, von Segesser LK. Optimized venous return with a self-expanding cannula: from computational fluid dynamics to clinical application. Interact Cardiovasc Thorac Surg. 2002; (1): 23-7. https://doi.org/10.1016/S1569-9293(02)00006-3.

12. Abdel-Sayed S, Abdel-Sayed P, Berdajs D, Ferrari E, Halbe M, von Segesser LK, et al. Effect of blood viscosity on the performance of virtually wall-less venous cannulas. Perfusion. 2019;35(5):393-6. https://doi.org/10.1177/02 67659119885514.

13. Mikael BL, Prahl WL, Jeker WC, et al. Pressure and flow properties of cannulae for extracorporeal membrane Oxygenation II: drainage (venous) Cannulae. Perfusion. 2019:34(IS):65-73.

14. Ferrari E, von Segesser LK, Berdajs D, Müller L, Halbe M, Maisano F. Clinical experience in minimally invasive cardiac surgery with virtually wall-less venous cannulas. Innovations. 2018;13(2):104-7. https://doi.org/10.1097/imi 0000000000000478.

15. von Segesser LK, Berdajs D, Abdel-Sayed S, Tozzi P, Ferrari E, Maisano F. New virtually wall-less cannulas designed for augmented venous drainage in minimally invasive cardiac surgery. Innovations. 2016;11(4):278-81. https:// doi.org/10.1097/imi.0000000000000283.

16. Goebel N, Bonte D, Salehi-Gilani S, Nagib R, Ursulescu A, Franke UFW. Minimally invasive access aortic arch surgery. Innovations. 2017;12(5):351-5. https://doi.org/10.1097/imi.0000000000000390.

17. Mikael BL, Prahl WL, Jeker WC, et al. Pressure and flow properties of cannulae for extracorporeal membrane Oxygenation I: return (arterial) Cannulae. Perfusion. 2019;34(IS):58-64.

\section{Publisher's Note}

Springer Nature remains neutral with regard to jurisdictional claims in published maps and institutional affiliations.

Ready to submit your research? Choose BMC and benefit from:

- fast, convenient online submission

- thorough peer review by experienced researchers in your field

- rapid publication on acceptance

- support for research data, including large and complex data types

- gold Open Access which fosters wider collaboration and increased citations

- maximum visibility for your research: over $100 \mathrm{M}$ website views per year

At $\mathrm{BMC}$, research is always in progress.

Learn more biomedcentral.com/submissions 